\title{
Exponential Stabilization of Impulsive Switched Systems with Time Delays Using Guaranteed Cost Control
}

\author{
Xiang Xie, ${ }^{1}$ Honglei Xu, ${ }^{1,2}$ and Rong Zhang ${ }^{3}$ \\ ${ }^{1}$ School of Energy and Power Engineering, Huazhong University of Science and Technology, Wuhan 430074, China \\ ${ }^{2}$ Department of Mathematics and Statistics, Curtin University, Perth, WA 6845, Australia \\ ${ }^{3}$ Huazhong University of Science and Technology Library, Wuhan 430074, China \\ Correspondence should be addressed to Rong Zhang; 82304356@qq.com
}

Received 21 March 2014; Accepted 28 April 2014; Published 7 May 2014

Academic Editor: Xinguang Zhang

Copyright (C) 2014 Xiang Xie et al. This is an open access article distributed under the Creative Commons Attribution License, which permits unrestricted use, distribution, and reproduction in any medium, provided the original work is properly cited.

\begin{abstract}
This paper investigates the stabilization problem for impulsive switched systems with time delays. First, exponential stability criteria of the delayed impulsive switched systems are established by use of the Lyapunov-Krasovskii functional method. Based on these results, sufficient conditions for the existence of a guaranteed cost control are also given. Subject to these sufficient conditions, the closed-loop impulsive switched system under the guaranteed cost control law will be exponentially stable with a guaranteed cost value.
\end{abstract}

\section{Introduction}

During the evolution process of a variety of disciplines such as engineering, economics, and biology, there often exists an impressive characteristic that the system state changes rapidly due to state jumping and the coexistence of continuous dynamics and discrete events. These jumping effects and switching phenomena can be modeled as an impulsive switched system. The system stability problem is one of main problems for such impulsive switched systems.

Recently, there are many results on stability analysis of impulsive switched systems. For instance, the robust stabilization problem for a class of impulsive switched systems under the LQ guaranteed cost control is studied in [1]. In addition, sufficient conditions for the existence of a guaranteed cost control law are given as well. In [2], sufficient conditions, independent of time delays and impulsive switching intervals, are derived by using a Lyapunov-Krasovskii's technique, ensuring asymptotical stability of impulsive switched systems with time invariant delays. Moreover, stability criteria problems for uncertain impulsive switched systems with input delay are studied by using the receding horizon method in [3]. Appropriate switching controllers are designed and linear matrix inequality conditions are derived to guarantee the closed-loop uncertain impulsive switched systems under a designed delayed controller. Other relevant references can be found in [4-9].

On the other hand, time delay has become a common phenomenon frequently occurred in science and engineering. Stability of time delay systems has received increasing attention among the applied mathematics and control community; for example, see [10-12] and the references therein. Most of these results are derived by use of either the Razumikhin method or the Lyapunov-Krasovskii method.

Since both impulses and time delays are ubiquitous in the real world, it is necessary to analyze their stability performance of dynamical systems involving impulses, time delays, and switchings. There already existed some effective results for the stability analysis of either impulsive switched systems or time delay systems mentioned above. However, there are few results on impulsive switched systems with time delays. This motivates our research in this paper. Our aim is to apply a set of improved Lyapunov functions to analyze the exponential stability performance of the impulsive switched systems with time delays, to derive sufficient conditions to ensure the exponential stability, and to design guaranteed cost controllers based on the linear matrix inequality approach. The designed guaranteed cost controller can ensure not only 
exponential stability of the closed-loop system but also the guaranteed cost performance.

The remaining sections of the paper proceed as follows. Section 2 provides necessary propositions and definitions. In Section 3, sufficient conditions for ensuring the closed-loop system exponentially stable are presented, and the designed guaranteed cost controller for impulsive switched systems with time delays are presented. Finally, conclusion remarks are presented in Section 4.

\section{Problem Statement}

Consider the following impulsive switched system with time delay:

$$
\begin{gathered}
\dot{x}(t)=\widehat{A}_{i_{k}} x(t)+\widehat{B}_{i_{k}} x(t-h(t))+C_{i_{k}} u(t) \quad t \neq t_{k}, \\
\Delta x(t)=D_{k} x(t) \quad t=t_{k}, \\
x(t)=\varphi(t) \quad-1 \leq t \leq 0, \\
\widehat{A}_{i_{k}}=A_{i_{k}}+\Delta A_{i_{k}}, \quad \widehat{B}_{i_{k}}=B_{i_{k}}+\Delta B_{i_{k}},
\end{gathered}
$$

where $x(t) \in R^{n}$ is the state, $u(t) \in R^{P}$ is the control input, $n, p \in N . A_{i_{k}}, B_{i_{k}}, C_{i_{k}}$, and $D_{k}$ are constant real matrices with appropriate dimensions. The delay function $h(t)$ satisfies the condition

$$
0 \leq h_{1} \leq h(t) \leq h_{2}
$$

$\Delta x(t)=x\left(t^{+}\right)-x\left(t^{-}\right), x\left(t^{+}\right)=\lim _{v \rightarrow 0^{+}} x(t+v), \lim _{v \rightarrow 0^{+}} x(t-$ $v)=x\left(t_{k}^{-}\right)=x\left(t_{k}\right)$, where $i_{k} \in\{1,2,3, \ldots, m\}, k \in N$, $m \in N$. $t_{k}$ is an impulsive switching point, $t_{0}<t_{1}<t_{2}<\cdots<$ $t_{k}<\cdots<t_{\infty} . \Delta A_{i_{k}}(\cdot)$ and $\Delta B_{i_{k}}(\cdot)$ are unknown real normbounded matrix valued functions, representing time-varying parameter uncertainties. The admissible uncertainties are assumed to be of the form

$$
\left[\Delta A_{i_{k}}(t) \quad \Delta B_{i_{k}}(t)\right]=E_{i_{k}} \Psi_{i_{k}}(t)\left[\begin{array}{ll}
H_{i_{k}} & J_{i_{k}}
\end{array}\right] .
$$

$E_{i_{k}}, H_{i_{k}}, J_{i_{k}}$ are known real constant matrices, $\Psi_{i_{k}}(t)$ is an unknown real time-varying matrix satisfying $\Psi_{i_{k}}^{T}(t) \Psi_{i_{k}}(t)<I$, and $I$ is the identity matrix with appropriate dimension.

Consider the following cost function:

$$
J=\sum_{k=0}^{\infty} \int_{t_{k}}^{t_{k+1}}\left[x^{T}(t) R_{i_{k}} x(t)+u^{T}(t) Q_{i_{k}} u(t)\right] d t
$$

where $R_{i_{k}}>0$ and $Q_{i_{k}}>0$ are positive definite symmetric matrices.

Definition 1. Suppose that there exist a control law $u(t)=$ $F_{i_{k}} x(t)$ and a positive constant $J^{*}$ such that, for all admissible uncertainties, the closed-loop system ((1a), (1b), (1c), and (1d)) is exponentially stable and the cost function (4) satisfies $J \leq J^{*}$, and then $u(t)$ is the exponential guaranteed cost control law for the impulsive switched system with time delay. Here, $J^{*}$ is the guaranteed cost value.
In addition, the following lemmas are necessary to proceed.

Lemma 2 (see [13, Proposition 2.2]). Let $A, D, E$, and $F$ be real matrices of appropriate dimensions with $\|F\| \leq 1$. Then, for any matrix $P=P^{T}>0$ and scalar $\varepsilon>0$,

$$
\begin{gathered}
\varepsilon I-E P E^{T}>0 \\
(A+D F E) P(A+D F E)^{T} \\
\leq A P A^{T}+A P E^{T}\left(\varepsilon I-E P E^{T}\right)^{-1} E P A^{T}+\varepsilon D D^{T} .
\end{gathered}
$$

Lemma 3 (Schur complement lemma (see [14])). Given constant matrices $X, Y$, and $Z$ with appropriate dimensions satisfying $X=X^{T}$ and $Y=Y^{T}>0$. then $X+Z^{T} Y^{-1} Z<0$ if and only if

$$
\left(\begin{array}{ll}
X & Z^{T} \\
Z & -Y
\end{array}\right)<0
$$

Lemma 4 (integral inequality [15]). For any constant matrix $Z=Z^{T}>0$ and a scalar $b$ such that the following integrations are well defined,

$$
b \int_{t-b}^{t} x(s)^{T} Z x(s) d s \geq\left(\int_{t-b}^{t} x(s) d s\right)^{T} Z\left(\int_{t-b}^{t} x(s) d s\right) .
$$

\section{Main Results}

First, we will present sufficient conditions for exponential stability of the impulsive switched system ((1a), (1b), (1c), and (1d)) without control input.

Theorem 5. Consider the time-delayed impulsive switched system ((1a), (1b), (1c), and (1d)) with the cost function (4). Suppose there exist $\alpha>0, \varepsilon_{1}>0, P_{i_{k}}=P_{i_{k}}^{T}>0$, such that the following conditions are satisfied:

(i)

$$
\left[\begin{array}{ccccc}
k_{11} & 0 & \lambda_{2} S_{1} & \lambda_{3} S_{2} & 0 \\
* & k_{22} & \lambda_{3} S_{3} & \lambda_{3} S_{3} & 0 \\
* & * & k_{33} & 0 & 0 \\
* & * & * & k_{44} & 0 \\
* & * & * & * & k_{55}
\end{array}\right]<0,
$$

$$
\left[\begin{array}{cc}
P_{i_{k-1}} & \left(I+D_{k}\right)^{T} P_{i_{k}} \\
P_{i_{k}}\left(I+D_{k}\right) & P_{i_{k}}
\end{array}\right]>0,
$$


where,

$$
\begin{aligned}
& k_{11}=\widehat{A}_{i_{k}}^{T} P_{i_{k}}+P_{i_{k}} \widehat{A}_{i_{k}}+U_{1}+U_{2}-\lambda_{2} S_{1}-\lambda_{3} S_{2}+2 \alpha P_{i_{k}}+R_{i_{k}}, \\
& k_{22}=\widehat{B}_{i_{k}}^{T} P_{i_{k}}+P_{i_{k}} \widehat{B}_{i_{k}}-2 \lambda_{3} S_{3}, \\
& k_{33}=-\lambda_{2} U_{1}-\lambda_{2} S_{1}-\lambda_{3} S_{3}, \\
& k_{44}=-\lambda_{3} U_{2}-\lambda_{3} S_{2}-\lambda_{3} S_{3}, \\
& k_{55}=h_{1}^{2} S_{1}+h_{2}^{2} S_{2}+\left(h_{2}-h_{1}\right)^{2} S_{3} .
\end{aligned}
$$

Then, the impulsive switched system ((1a), (1b), (1c), and (1d)) is exponentially stable.

Proof. When $t \in\left(t_{k}, t_{k+1}\right]$, consider the Lyapunov-Krasovskii function candidate

$$
V\left(t, x_{t}\right)=\sum_{i=1}^{4} V_{i}\left(t, x_{t}\right)
$$

where

$$
\begin{gathered}
V_{1}\left(t, x_{t}\right)=x^{T}(t) P_{i_{k}} x(t), \\
V_{2}\left(t, x_{t}\right)=\int_{t-h_{1}}^{t} e^{2 \alpha(s-t)} x^{T}(s) U_{1} x(s) d s \\
+\int_{t-h_{2}}^{t} e^{2 \alpha(s-t)} x^{T}(s) U_{2} x(s) d s, \\
V_{3}\left(t, x_{t}\right)=h_{1} \int_{-h_{1}}^{0} \int_{t+s}^{t} e^{2 \alpha(\tau-s)} \dot{x}^{T}(\tau) S_{1} \dot{x}(\tau) d \tau d s \\
+h_{2} \int_{-h_{2}}^{0} \int_{t+s}^{t} e^{2 \alpha(\tau-s)} \dot{x}^{T}(\tau) S_{2} \dot{x}(\tau) d \tau d s, \\
V_{4}\left(t, x_{t}\right)= \\
\left(h_{2}-h_{1}\right) \\
\\
\times \int_{-h_{2}}^{-h_{1}} \int_{t+s}^{t} e^{2 \alpha(\tau-s)} \dot{x}^{T}(\tau) S_{3} \dot{x}(\tau) d \tau d s .
\end{gathered}
$$

Let

$$
\begin{aligned}
\Omega= & \lambda_{\max }\left(P_{i_{k}}\right)+h_{1} \lambda_{\max }\left(U_{1}\right)+h_{2} \lambda_{\max }\left(U_{2}\right) \\
& +0.5 h_{1}^{3} \lambda_{\max }\left(S_{1}\right)+0.5 h_{2}^{3} \lambda_{\max }\left(S_{2}\right) \\
& +\left(h_{2}^{2}-h_{1}^{2}\right)\left(h_{2}-h_{1}\right) \lambda_{\max }\left(S_{3}\right) ;
\end{aligned}
$$

then, we can easily check that

$$
\lambda\|x(t)\|^{2} \leq V\left(t, x_{t}\right) \leq \Omega\|x(t)\|^{2}, \quad t \in R^{+} .
$$

Taking the derivative of $V_{i}\left(t, x_{t}\right), i=1,2,3,4$, along the trajectory of the closed-loop system, we obtain

$$
\begin{aligned}
\dot{V}_{1}\left(t, x_{t}\right) & \\
= & {\left[\widehat{A}_{i_{k}} x(t)+\widehat{B}_{i_{k}} x(t-h(t))\right]^{T} P_{i_{k}} x(t) } \\
& +x^{T}(t) P_{i_{k}}\left[\widehat{A}_{i_{k}} x(t)+\widehat{B}_{i_{k}} x(t-h(t))\right] \\
= & x^{T}(t)\left[\widehat{A}_{i_{k}}^{T} P_{i_{k}}+P_{i_{k}} \widehat{A}_{i_{k}}\right] x(t)
\end{aligned}
$$

$$
+x^{T}(t-h(t)) \widehat{B}_{i_{k}}^{T} P_{i_{k}} x(t)
$$

$$
+x^{T}(t) P_{i_{k}} \widehat{B}_{i_{k}}^{T} x(t-h(t)),
$$

$\dot{V}_{2}\left(t, x_{t}\right)$

$$
\begin{aligned}
=-2 \alpha e^{-2 \alpha t}\left[\int_{t-h_{1}}^{t} e^{2 \alpha s} x^{T}(s) U_{1} x(s) d s\right. \\
\left.+\int_{t-h_{2}}^{t} e^{2 \alpha s} x^{T}(s) U_{2} x(s) d s\right]
\end{aligned}
$$$$
+e^{-2 \alpha t}\left[e^{2 \alpha t} x^{T}(t) U_{1} x(t)-e^{2 \alpha\left(t-h_{1}\right)} x^{T}\left(t-h_{1}\right)\right.
$$$$
\times U_{1} x\left(t-h_{1}\right)+e^{2 \alpha t} x^{T}(t) U_{2} x(t)
$$

$$
\left.-e^{2 \alpha\left(t-h_{2}\right)} x^{T}\left(t-h_{2}\right) U_{1} x\left(t-h_{2}\right)\right]
$$

$$
=-2 \alpha V_{2}+x^{T}(t)\left(U_{1}+U_{2}\right) x(t)
$$$$
-\lambda_{2} x^{T}\left(t-h_{1}\right) U_{1} x\left(t-h_{1}\right)
$$$$
-\lambda_{3} x^{T}\left(t-h_{2}\right) U_{1} x\left(t-h_{2}\right),
$$

$$
\begin{aligned}
& \dot{V}_{3}\left(t, x_{t}\right) \\
& =-2 \alpha V_{3} \\
& \quad+e^{-2 \alpha t} \frac{d}{d t}\left[h_{1} \int_{-h_{1}}^{0} \int_{t+s}^{t} e^{2 \alpha \tau} \dot{x}^{T}(\tau) S_{1} \dot{x}(\tau) d \tau d s\right. \\
& \left.\quad+h_{2} \int_{-h_{2}}^{0} \int_{t+s}^{t} e^{2 \alpha \tau} \dot{x}^{T}(\tau) S_{2} \dot{x}(\tau) d \tau d s\right] \\
& =-2 \alpha V_{3}+\dot{x}^{T}(t)\left(h_{1}^{2} S_{1}+h_{2}^{2} S_{2}\right) \dot{x}(t) \\
& \quad-h_{1} \int_{-h_{1}}^{0} e^{2 \alpha s} \dot{x}^{T}(t+s) S_{1} \dot{x}(t+s) d s \\
& \quad-h_{2} \int_{-h_{2}}^{0} e^{2 \alpha s} \dot{x}^{T}(t+s) S_{2} \dot{x}(t+s) d s .
\end{aligned}
$$


From Lemma 4, we have

$$
\begin{aligned}
& \dot{V}_{3}\left(t, x_{t}\right) \\
& \leq-2 \alpha V_{3}+\dot{x}^{T}(t)\left(h_{1}^{2} S_{1}+h_{2}^{2} S_{2}\right) \dot{x}(t) \\
& -x^{T}(t)\left(\lambda_{2} S_{1}+\lambda_{3} S_{2}\right) x(t)+2 \lambda_{2} x^{T}(t) S_{1} x\left(t-h_{1}\right) \\
& -\lambda_{2} x^{T}\left(t-h_{1}\right) S_{1} x\left(t-h_{1}\right)+2 \lambda_{3} x^{T}(t) S_{2} x\left(t-h_{2}\right) \\
& -\lambda_{3} x^{T}\left(t-h_{2}\right) S_{2} x\left(t-h_{2}\right) \text {, } \\
& \dot{V}_{4}\left(t, x_{t}\right) \\
& =-2 \alpha V_{4}+e^{-2 \alpha t}\left(h_{2}-h_{1}\right) \\
& \times \int_{-h_{2}}^{-h_{1}}\left[e^{2 \alpha t} \dot{x}^{T}(t) S_{3} \dot{x}(t)\right. \\
& \left.-e^{2 \alpha(t+s)} \dot{x}^{T}(t+s) S_{3} \dot{x}(t+s)\right] d s \\
& =-2 \alpha V_{4}+\left(h_{2}-h_{1}\right)^{2} \dot{x}^{T}(t) S_{3} \dot{x}(t) \\
& -\left(h_{2}-h_{1}\right) \int_{-h_{2}}^{-h_{1}} e^{2 \alpha s} \dot{x}^{T}(t+s) S_{3} \dot{x}(t+s) d s \\
& \leq-2 \alpha V_{4}+\left(h_{2}-h_{1}\right)^{2} \dot{x}^{T}(t) S_{3} \dot{x}(t) \\
& -\left(h_{2}-h_{1}\right) e^{-2 \alpha h_{2}}\left[\int_{-h_{2}}^{-h} \dot{x}^{T}(t+s) S_{3} \dot{x}(t+s) d s\right. \\
& \left.+\int_{-h}^{-h_{1}} \dot{x}^{T}(t+s) S_{3} \dot{x}(t+s) d s\right] \\
& \leq-2 \alpha V_{4}+\left(h_{2}-h_{1}\right)^{2} \dot{x}^{T}(t) S_{3} \dot{x}(t) \\
& -\lambda_{3}\left[2 x^{T}(t-h(t)) S_{3} x(t-h(t))\right. \\
& -2 x^{T}(t-h(t)) S_{3} x\left(t-h_{2}\right) \\
& +x^{T}\left(t-h_{2}\right) S_{3} x\left(t-h_{2}\right) \\
& +x^{T}\left(t-h_{1}\right) S_{3} x\left(t-h_{1}\right) \\
& \left.-2 x^{T}(t-h(t)) S_{3} x\left(t-h_{1}\right)\right] \text {. }
\end{aligned}
$$

$$
\begin{aligned}
& +\dot{x}^{T}(t)\left[h_{1}^{2} S_{1}+h_{2}^{2} S_{2}+\left(h_{2}-h_{1}\right)^{2} S_{3}\right] \dot{x}(t) \\
& -x^{T}(t) R_{i_{k}} x(t) .
\end{aligned}
$$

Let $\xi^{T}(t)=\left[x^{T}(t), x^{T}(t-h(t)), x^{T}\left(t-h_{1}\right), x^{T}\left(t-h_{2}\right), \dot{x}^{T}(t)\right]$,

$$
k=\left[\begin{array}{ccccc}
k_{11} & 0 & \lambda_{2} S_{1} & \lambda_{3} S_{2} & 0 \\
* & k_{22} & \lambda_{3} S_{3} & \lambda_{3} S_{3} & 0 \\
* & * & k_{33} & 0 & 0 \\
* & * & * & k_{44} & 0 \\
* & * & * & * & k_{55}
\end{array}\right] .
$$

We have

$$
\dot{V}\left(t, x_{t}\right)+2 \alpha V\left(t, x_{t}\right) \leq \xi^{T}(t) k \xi(t)-x^{T}(t) R_{i_{k}} x(t) .
$$

Since $k<0$ from ((1a), (1b), (1c), and (1d)), it is clear that $\dot{V}\left(t, x_{t}\right)+2 \alpha V\left(t, x_{t}\right)<0$ or, equivalently, $V\left(t, x_{t}\right) \leq$ $V\left(0, x_{0}\right) e^{-2 \alpha t}, \forall t \in R^{+}$.

Taking (14) into account, we have

$$
\lambda\|x(t)\|^{2} \leq V\left(t, x_{t}\right) \leq V\left(0, x_{0}\right) e^{-2 \alpha t} \leq \Omega e^{-2 \alpha t}\|x(0)\|^{2},
$$

and hence

$$
\|x(t)\| \leq \sqrt{\frac{\Omega}{\lambda}}\|x(0)\| e^{-\alpha t},
$$

which shows that the impulsive switched system ((1a), (1b), (1c), and (1d)) is exponentially stable when $t \in\left(t_{k}, t_{k+1}\right]$.

Next, let us consider the case at the impulsive and switching time point $t_{k}, t_{k}=1,2,3, \ldots$,

$$
V\left(t_{k}^{+}\right)-V\left(t_{k}^{-}\right)=x\left(t_{k}^{+}\right)^{T} P_{i_{k}} x\left(t_{k}^{+}\right)-x\left(t_{k}^{-}\right)^{T} P_{i_{k-1}} x\left(t_{k}^{-}\right) .
$$

Noting that $\Delta x\left(t_{k}\right)=x\left(t_{k}^{+}\right)-x\left(t_{k}^{-}\right), x\left(t_{k}^{-}\right)=x\left(t_{k}\right)$, we have

$$
\begin{aligned}
& V\left(t_{k}^{+}\right)-V\left(t_{k}^{-}\right) \\
& \quad=x\left(t_{k}^{+}\right)^{T} P_{i_{k}} x\left(t_{k}^{+}\right)-x\left(t_{k}\right)^{T} P_{i_{k-1}} x\left(t_{k}\right) \\
& \quad=x\left(t_{k}\right)^{T}\left[\left(I+D_{k}\right)^{T} P_{i_{k}}\left(I+D_{k}\right)-P_{i_{k-1}}\right] x\left(t_{k}\right) .
\end{aligned}
$$

From (ii), we know that $\left[\begin{array}{cc}P_{i_{k-1}} & \left(I+D_{k}\right)^{T} P_{i_{k}} \\ P_{i_{k}}\left(I+D_{k}\right) & P_{i_{k}}\end{array}\right]>0$, which is equivalent to $\left(I+D_{k}\right)^{T} P_{i_{k}}\left(I+D_{k}\right)-P_{i_{k-1}}<0$. 
So, $V\left(t_{k}^{+}\right)-V\left(t_{k}^{-}\right)<0$. Therefore, we can conclude that the impulsive switched system ((1a), (1b), (1c), and (1d)) is exponentially stable.

Theorem 5 develops sufficient conditions for exponential stability of the impulsive switched system ((1a), (1b), (1c), and (1d)) under the case that $u(t)=0$. In the next theorem, we will design the guaranteed cost controller in the form of $u(t)=F_{i_{k}} x(t)$ and sufficient conditions for the existence of a guaranteed cost control are also presented as well.

Theorem 6. Consider the impulsive switched system with time delay ((1a), (1b), (1c), and (1d)) with the cost function (4) and suppose there exist $\alpha>0, \varepsilon_{1}>0, P_{i_{k}}=P_{i_{k}}^{T}>0$. If there exist symmetric positive definite matrices $U_{1}, U_{2}, S_{1}, S_{2}, S_{3}$, and $K$ such that the following conditions are satisfied:

(i)

$$
\left[\begin{array}{cc}
\varepsilon_{1} I & E_{i_{k}}^{T} P_{i_{k}} \\
P_{i_{k}} E_{i_{k}} & P_{i_{k}}
\end{array}\right]>0,
$$

(ii)

(iii)

$$
\left[\begin{array}{cc}
P_{i_{k-1}} & \left(I+D_{k}\right)^{T} P_{i_{k}} \\
P_{i_{k}}\left(I+D_{k}\right) & P_{i_{k}}
\end{array}\right]>0,
$$

where

$$
\begin{gathered}
\Pi_{1}=\left[\begin{array}{ccccc}
\widetilde{w_{11}} & 0 & \lambda_{2} S_{1} & \lambda_{3} S_{2} & 0 \\
* & \widetilde{w_{22}} & \lambda_{3} S_{3} & \lambda_{3} S_{3} & 0 \\
* & * & w_{33} & 0 & 0 \\
* & * & * & w_{44} & 0 \\
* & * & * & * & w_{55}
\end{array}\right], \\
\Pi_{2}=\left[\begin{array}{ccccc}
A_{i_{k}}^{T} E_{i_{k}} & K & K\left(C_{i_{k}}^{-1}\right)^{T} & H_{i_{k}}^{T} & 0 \\
0 & 0 & 0 & 0 & \left(B_{i_{k}}+\Delta B_{i_{k}}\right)^{T} \\
0 & 0 & 0 & 0 & 0 \\
0 & 0 & 0 & 0 & 0 \\
0 & 0 & 0 & 0 & 0
\end{array}\right], \\
\widetilde{w_{11}}=A_{i_{k}}^{T} A_{i_{k}}+3 P_{i_{k}}^{2}+U_{1}+U_{2}-\lambda_{2} S_{1}-\lambda_{3} S_{2}+2 \alpha P_{i_{k}}+R_{i_{k}},
\end{gathered}
$$

$$
\begin{gathered}
\widetilde{w_{22}}=-2 \lambda_{3} S_{3}, \\
w_{33}=-\lambda_{2} U_{1}-\lambda_{2} S_{1}-\lambda_{3} S_{3}, \\
w_{44}=-\lambda_{3} U_{2}-\lambda_{3} S_{2}-\lambda_{3} S_{3}, \\
w_{55}=h_{1}^{2} S_{1}+h_{2}^{2} S_{2}+\left(h_{2}-h_{1}\right)^{2} S_{3}, \\
\lambda_{1}=e^{-2 \alpha\left(h_{1}+h_{2}\right)}, \quad \lambda_{2}=e^{-2 \alpha h_{1}}, \\
\lambda_{3}=e^{-2 \alpha h_{2}}, \quad \lambda=\lambda_{\min }\left(P_{i_{k}}\right),
\end{gathered}
$$

then the guaranteed cost control law $u(t)=C_{i_{k}}^{-1} K^{T} x(t)$ can ensure that the impulsive switched system ((1a), (1b), (1c), and (1d)) is exponentially stable. Moreover, the guaranteed cost value is

$$
J^{*}=V\left(0, x_{0}\right) .
$$

Proof. When $t \in\left(t_{k}, t_{k+1}\right]$, choose the Lyapunov-Krasovskii function candidate to be similar to Theorem 5

$$
V\left(t, x_{t}\right)=\sum_{i=1}^{4} V_{i}\left(t, x_{t}\right)
$$

where

$$
V_{1}\left(t, x_{t}\right)=x^{T}(t) P_{i_{k}} x(t),
$$

$$
\begin{aligned}
V_{2}\left(t, x_{t}\right)= & \int_{t-h_{1}}^{t} e^{2 \alpha(s-t)} x^{T}(s) U_{1} x(s) d s \\
& +\int_{t-h_{2}}^{t} e^{2 \alpha(s-t)} x^{T}(s) U_{2} x(s) d s,
\end{aligned}
$$

$$
\begin{aligned}
V_{3}\left(t, x_{t}\right)= & h_{1} \int_{-h_{1}}^{0} \int_{t+s}^{t} e^{2 \alpha(\tau-s)} \dot{x}^{T}(\tau) S_{1} \dot{x}(\tau) d \tau d s \\
& +h_{2} \int_{-h_{2}}^{0} \int_{t+s}^{t} e^{2 \alpha(\tau-s)} \dot{x}^{T}(\tau) S_{2} \dot{x}(\tau) d \tau d s, \\
V_{4}\left(t, x_{t}\right)= & \left(h_{2}-h_{1}\right) \\
& \times \int_{-h_{2}}^{-h_{1}} \int_{t+s}^{t} e^{2 \alpha(\tau-s)} \dot{x}^{T}(\tau) S_{3} \dot{x}(\tau) d \tau d s .
\end{aligned}
$$

Taking the derivative of $V_{i}\left(t, x_{t}\right), i=1,2,3,4$, along the trajectory of the closed-loop system, we obtain

$$
\begin{aligned}
\dot{V}_{1}\left(t, x_{t}\right) & \\
= & {\left[\widehat{A}_{i_{k}} x(t)+\widehat{B}_{i_{k}} x(t-h(t))+C_{i_{k}} u(t)\right]^{T} P_{i_{k}} x(t) } \\
& +x^{T}(t) P_{i_{k}}\left[\widehat{A}_{i_{k}} x(t)+\widehat{B}_{i_{k}} x(t-h(t))+C_{i_{k}} u(t)\right] .
\end{aligned}
$$


Put (1d) and $u(t)=F_{i_{k}} x(t)$ into it and then we can get

$$
\begin{gathered}
\dot{V}_{1}\left(t, x_{t}\right)=x^{T}(t)\left[\left(A_{i_{k}}+\Delta A_{i_{k}}\right)^{T} P_{i_{k}}+P_{i_{k}}\left(A_{i_{k}}+\Delta A_{i_{k}}\right)\right. \\
\left.+F_{i_{k}}^{T} C_{i_{k}}^{T} P_{i_{k}}+P_{i_{k}} C_{i_{k}} F_{i_{k}}\right] x(t) \\
+x^{T}(t-h(t))\left(B_{i_{k}}+\Delta B_{i_{k}}\right)^{T} P_{i_{k}} x(t) \\
+x^{T}(t) P_{i_{k}}\left(B_{i_{k}}+\Delta B_{i_{k}}\right) x(t-h(t))
\end{gathered}
$$

Next, it follows from Lemma 2 that

$$
\begin{aligned}
\left(A_{i_{k}}+\Delta A_{i_{k}}\right)^{T} P_{i_{k}}+P_{i_{k}}\left(A_{i_{k}}+\Delta A_{i_{k}}\right) \\
\leq\left(A_{i_{k}}+\Delta A_{i_{k}}\right)^{T}\left(A_{i_{k}}+\Delta A_{i_{k}}\right)+P_{i_{k}}^{2} \\
\leq A_{i_{k}}^{T} A_{i_{k}}+A_{i_{k}}^{T} E_{i_{k}}\left(\varepsilon_{1} I-E_{i_{k}}^{T} E_{i_{k}}\right)^{-1} E_{i_{k}}^{T} A_{i_{k}} \\
\quad+\varepsilon_{1} H_{i_{k}}^{T} H_{i_{k}}+P_{i_{k}}^{2}, \\
x^{T}(t-h(t))\left(B_{i_{k}}+\Delta B_{i_{k}}\right)^{T} P_{i_{k}} x(t) \\
\quad+x^{T}(t) P_{i_{k}}\left(B_{i_{k}}+\Delta B_{i_{k}}\right) x(t-h(t)) \\
\leq x^{T}(t) P_{i_{k}}^{2} x(t)+x^{T}(t-h(t)) \\
\quad \times\left(B_{i_{k}}+\Delta B_{i_{k}}\right)^{T}\left(B_{i_{k}}+\Delta B_{i_{k}}\right) x(t-h(t)) .
\end{aligned}
$$

Substituting (36) into (35) yields

$$
\begin{aligned}
\dot{V}_{1}\left(t, x_{t}\right) \leq & x^{T}(t)\left[A_{i_{k}}^{T} A_{i_{k}}+A_{i_{k}}^{T} E_{i_{k}}\left(\varepsilon_{1} I-E_{i_{k}}^{T} E_{i_{k}}\right)^{-1} E_{i_{k}}^{T} A_{i_{k}}\right. \\
& \left.+\varepsilon_{1} H_{i_{k}}^{T} H_{i_{k}}+3 P_{i_{k}}^{2}+F_{i_{k}}^{T} C_{i_{k}}^{T} C_{i_{k}} F_{i_{k}}\right] x(t) \\
& +x^{T}(t-h(t))\left[\left(B_{i_{k}}+\Delta B_{i_{k}}\right)^{T}\left(B_{i_{k}}+\Delta B_{i_{k}}\right)\right] \\
& \times x(t-h(t)) .
\end{aligned}
$$

The derivatives of $V_{2}\left(t, x_{t}\right), V_{3}\left(t, x_{t}\right)$, and $V_{4}\left(t, x_{t}\right)$ are the same as those in Theorem 5. Then, we can put (37), (16), (18), and (19) together to obtain

$$
\begin{aligned}
& \dot{V}\left(t, x_{t}\right)+ 2 \alpha V\left(t, x_{t}\right) \\
& \leq x^{T}(t)\left[A_{i_{k}}^{T} A_{i_{k}}+A_{i_{k}}^{T} E_{i_{k}}\left(\varepsilon_{1} I-E_{i_{k}}^{T} E_{i_{k}}\right)^{-1} E_{i_{k}}^{T} A_{i_{k}}\right. \\
&+\varepsilon_{1} H_{i_{k}}^{T} H_{i_{k}}+3 P_{i_{k}}^{2}+F_{i_{k}}^{T} C_{i_{k}}^{T} C_{i_{k}} F_{i_{k}}+U_{1} \\
&+U_{2}-\lambda_{2} S_{1}-\lambda_{3} S_{2}+2 \alpha P_{i_{k}}+R_{i_{k}} \\
&\left.+F_{i_{k}}^{T} Q_{i_{k}} F_{i_{k}}\right] x(t)
\end{aligned}
$$

$$
\begin{aligned}
& +x^{T}(t-h(t))\left[\left(B_{i_{k}}+\Delta B_{i_{k}}\right)^{T}\left(B_{i_{k}}+\Delta B_{i_{k}}\right)-2 \lambda_{3} S_{3}\right] \\
& \times x(t-h(t)) \\
& +x^{T}\left(t-h_{1}\right)\left[-\lambda_{2} U_{1}-\lambda_{2} S_{1}-\lambda_{3} S_{3}\right] x\left(t-h_{1}\right) \\
& +x^{T}\left(t-h_{2}\right)\left[-\lambda_{3} U_{2}-\lambda_{3} S_{2}-\lambda_{3} S_{3}\right] x\left(t-h_{2}\right) \\
& +2 \lambda_{3} x^{T}(t) S_{2} x\left(t-h_{2}\right)+2 \lambda_{2} x^{T}(t) S_{1} x\left(t-h_{1}\right) \\
& +2 \lambda_{3} x^{T}(t-h(t)) S_{3} x\left(t-h_{2}\right) \\
& +2 \lambda_{3} x^{T}(t-h(t)) S_{3} x\left(t-h_{1}\right) \\
& +\dot{x}^{T}(t)\left[h_{1}^{2} S_{1}+h_{2}^{2} S_{2}+\left(h_{2}-h_{1}\right)^{2} S_{3}\right] \dot{x}(t) \\
& -x^{T}(t)\left[R_{i_{k}}+F_{i_{k}}^{T} Q_{i_{k}} F_{i_{k}}\right] x(t) .
\end{aligned}
$$

Let

$$
\begin{gathered}
w=\left[\begin{array}{ccccc}
w_{11} & 0 & \lambda_{2} S_{1} & \lambda_{3} S_{2} & 0 \\
* & w_{22} & \lambda_{3} S_{3} & \lambda_{3} S_{3} & 0 \\
* & * & w_{33} & 0 & 0 \\
* & * & * & w_{44} & 0 \\
* & * & * & * & w_{55}
\end{array}\right] \\
w_{11}=A_{i_{k}}^{T} A_{i_{k}}+A_{i_{k}}^{T} E_{i_{k}}\left(\varepsilon_{1} I-E_{i_{k}}^{T} E_{i_{k}}\right)^{-1} E_{i_{k}}^{T} A_{i_{k}} \\
+\varepsilon_{1} H_{i_{k}}^{T} H_{i_{k}}+3 P_{i_{k}}^{2}+F_{i_{k}}^{T} C_{i_{k}}^{T} C_{i_{k}} F_{i_{k}}+U_{1} \\
+U_{2}-\lambda_{2} S_{1}-\lambda_{3} S_{2}+2 \alpha P_{i_{k}}+R_{i_{k}}+F_{i_{k}}^{T} Q_{i_{k}} F_{i_{k}}, \\
w_{22}=\left(B_{i_{k}}+\Delta B_{i_{k}}\right)^{T}\left(B_{i_{k}}+\Delta B_{i_{k}}\right)-2 \lambda_{3} S_{3}, \\
w_{33}=-\lambda_{2} U_{1}-\lambda_{2} S_{1}-\lambda_{3} S_{3}, \\
w_{44}=-\lambda_{3} U_{2}-\lambda_{3} S_{2}-\lambda_{3} S_{3}, \\
w_{55}=h_{1}^{2} S_{1}+h_{2}^{2} S_{2}+\left(h_{2}-h_{1}\right)^{2} S_{3} .
\end{gathered}
$$

Then, we have

$$
\begin{aligned}
& \dot{V}\left(t, x_{t}\right)+2 \alpha V\left(t, x_{t}\right) \\
& \quad \leq \xi^{T}(t) w \xi(t)-x^{T}(t)\left[R_{i_{k}}+F_{i_{k}}^{T} Q_{i_{k}} F_{i_{k}}\right] x(t) .
\end{aligned}
$$

By Lemma 3, it proves that $w<0$ holds if

$$
\left[\begin{array}{ll}
w_{1} & w_{2} \\
* & w_{3}
\end{array}\right]<0,
$$


where

$$
\begin{gathered}
w_{1}=\left[\begin{array}{ccccc}
\widetilde{w_{11}} & 0 & \lambda_{2} S_{1} & \lambda_{3} S_{2} & 0 \\
* & \widetilde{w_{22}} & \lambda_{3} S_{3} & \lambda_{3} S_{3} & 0 \\
* & * & w_{33} & 0 & 0 \\
* & * & * & w_{44} & 0 \\
* & * & * & * & w_{55}
\end{array}\right], \\
w_{2}=\left[\begin{array}{ccccc}
A_{i_{k}}^{T} E_{i_{k}} & F_{i_{k}}^{T} C_{i_{k}}^{T} & F_{i_{k}}^{T} & H_{i_{k}}^{T} & 0 \\
0 & 0 & 0 & 0 & \left(B_{i_{k}}+\Delta B_{i_{k}}\right)^{T} \\
0 & 0 & 0 & 0 & 0 \\
0 & 0 & 0 & 0 & 0 \\
0 & 0 & 0 & 0 & 0
\end{array}\right], \\
w_{3}=\operatorname{diag}\left(E_{i_{k}}^{T} E_{i_{k}}-\varepsilon_{1} I,-I,-Q_{i_{k}}^{-1},-\frac{1}{\varepsilon_{1}} I,-I\right), \\
\widetilde{w_{11}}=A_{i_{k}}^{T} A_{i_{k}}+3 P_{i_{k}}^{2}+U_{1}+U_{2} \\
-\lambda_{2} S_{1}-\lambda_{3} S_{2}+2 \alpha P_{i_{k}}+R_{i_{k}}, \\
\widetilde{w_{22}}=-2 \lambda_{3} S_{3} .
\end{gathered}
$$

Letting $F_{i_{k}}^{T} C_{i_{k}}^{T}=K$, we get $F_{i_{k}}=C_{i_{k}}^{-1} K^{T}$. Then, we have

$$
\begin{gathered}
{\left[\begin{array}{cc}
w_{1} & w_{2} \\
* & w_{3}
\end{array}\right]=\left[\begin{array}{cc}
\Pi_{1} & \Pi_{2} \\
* & \Pi_{3}
\end{array}\right]<0,} \\
\Pi_{1}=w_{1}, \\
\Pi_{2}=\left[\begin{array}{ccccc}
A_{i_{k}}^{T} E_{i_{k}} & K & K\left(C_{i_{k}}^{-1}\right)^{T} & H_{i_{k}}^{T} & 0 \\
0 & 0 & 0 & 0 & \left(B_{i_{k}}+\Delta B_{i_{k}}\right)^{T} \\
0 & 0 & 0 & 0 & 0 \\
0 & 0 & 0 & 0 & 0 \\
0 & 0 & 0 & 0 & 0
\end{array}\right] \\
\Pi_{3}=w_{3} .
\end{gathered}
$$

In addition, since $\left[\begin{array}{cc}\Pi_{1} & \Pi_{2} \\ * & \Pi_{3}\end{array}\right]<0$ (see Theorem 6 (ii)), $\left[\begin{array}{cc}w_{1} & w_{2} \\ * & w_{3}\end{array}\right]<$ 0 , then we plug it into (40) and obtain

$$
\dot{V}\left(t, x_{t}\right)+2 \alpha V\left(t, x_{t}\right)<0 \text {. }
$$

Now, we can easily conclude that the impulsive switched system ((1a), (1b), (1c), and (1d)) is exponentially stable. Looking back to (40), we can get

$$
\begin{aligned}
& \dot{V}\left(t, x_{t}\right) \leq-x^{T}(t)\left[R_{i_{k}}+F_{i_{k}}^{T} Q_{i_{k}} F_{i_{k}}\right] x(t), \\
& J=\sum_{k=0}^{\infty} \int_{t_{k}}^{t_{k+1}}\left[x^{T}(t) R_{i_{k}} x(t)+u^{T}(t) Q_{i_{k}} u(t)\right] d t \\
& \leq-\lim _{k \rightarrow \infty} \sum_{j=0}^{k} \int_{t_{j}}^{t_{j+1}} \frac{d V\left(t, x_{t}\right)}{d t} d t \\
& =-\lim _{k \rightarrow \infty}\left\{-V\left(0, x_{0}\right)+V\left(t_{1}^{-}, x_{t_{1}^{-}}\right)-V\left(t_{1}^{+}, x_{t_{1}^{+}}\right)\right. \\
& +V\left(t_{2}^{-}, x_{t_{2}^{-}}\right)-V\left(t_{2}^{+}, x_{t_{2}^{+}}\right) \\
& +\cdots-V\left(t_{k}^{-}, x_{t_{k}^{-}}\right) \\
& \left.+V\left(t_{k}^{+}, x_{t_{k}^{+}}\right)-V\left(t_{k+1}^{-}, x_{t_{k+1}^{-}}\right)\right\} \\
& =\lim _{k \rightarrow \infty}\left\{V\left(0, x_{0}\right)+\sum_{j=1}^{k}\left\{V\left(t_{j}^{+}, x_{t_{j}^{+}}\right)-V\left(t_{j}^{-}, x_{t_{j}^{-}}\right)\right\}\right. \\
& \left.+V\left(t_{k+1}^{-}, x_{t_{k+1}^{-}}\right)\right\} \\
& =V\left(0, x_{0}\right) \\
& +\lim _{k \rightarrow \infty} \sum_{j=1}^{k} x\left(t_{j}\right)^{T}\left\{\left(I+D_{j}\right)^{T} P_{i_{j}}\left(I+D_{j}\right)-P_{i_{j-1}}\right\} x\left(t_{j}\right) \\
& +\lim _{k \rightarrow \infty} V\left(t_{k+1}^{-}, x_{t_{k+1}^{-}}\right) \text {. }
\end{aligned}
$$

We know that

$$
\begin{gathered}
\lim _{k \rightarrow \infty} V\left(t_{k+1}^{-}, x_{t_{k+1}^{-}}\right)=0, \\
\lim _{k \rightarrow \infty} \sum_{j=1}^{k} x\left(t_{j}\right)^{T}\left\{\left(I+D_{j}\right)^{T} P_{i_{j}}\left(I+D_{j}\right)-P_{i_{j-1}}\right\} x\left(t_{j}\right)<0 .
\end{gathered}
$$

Thus, we can get

$$
J<V\left(0, x_{0}\right)=J^{*} .
$$

This completes the proof.

\section{Conclusion}

The exponential stabilization problem for impulsive switched systems with time delays is considered in this paper. Sufficient conditions for exponential stability of the impulsive switched systems without control input are derived via the LyapunovKrasovskii functional method. Moreover, sufficient conditions for the exponential stability of the impulsive switched 
systems under the designed guaranteed cost control law are established and the corresponding guaranteed cost value can be obtained as well.

\section{Conflict of Interests}

The authors declare that there is no conflict of interests regarding the publication of this paper.

\section{Acknowledgments}

This work is partially supported by the National Natural Science Foundation of China (nos. 11171079, 61290325, and 61321003), HUST Startup Research Fund, and HUST Independent Innovation Research Fund (GF and Natural Science).

\section{References}

[1] H. Xu, K. L. Teo, and X. Liu, "Robust stability analysis of guaranteed cost control for impulsive switched systems," IEEE Translations on Systems, Man, and Cybernetics B, vol. 38, pp. 1419-1422, 2008.

[2] H. Xu, X. Liu, and K. L. Teo, "A LMI approach to stability analysis and synthesis of impulsive switched systems with time delays," Nonlinear Analysis: Hybrid Systems, vol. 2, no. 1, pp. 3850, 2008.

[3] H. Xu and K. L. Teo, "Robust stabilization of uncertain impulsive switched systems with delayed control," Computers \& Mathematics with Applications, vol. 56, no. 1, pp. 63-70, 2008.

[4] H. Xu, X. Liu, and K. L. Teo, "Delay independent stability criteria of impulsive switched systems with time-invariant delays," Mathematical and Computer Modelling, vol. 47, no. 3-4, pp. 372379, 2008.

[5] P. Niamsup, "Stability of time-varying switched systems with time-varying delay," Nonlinear Analysis: Hybrid Systems, vol. 3, no. 4, pp. 631-639, 2009.

[6] G. Garcia, B. Pradin, S. Tarbouriech, and F. Zeng, "Robust stabilization and guaranteed cost control for discrete-time linear systems by static output feedback," Automatica, vol. 39, no. 9, pp. 1635-1641, 2003.

[7] H. Xu and K. L. Teo, " $H_{\infty}$ optimal stabilization of a class of uncertain impulsive systems: an LMI approach," Journal of Industrial and Management Optimization, vol. 5, no. 1, pp. 153159, 2009.

[8] Y. Zhang, J. Yang, H. Xu, and K. L. Teo, "New results on practical set stability of switched nonlinear systems," in Proceedings of the 3rd Australian Control Conference (AUCC '13), pp. 164-168, 2013.

[9] Y. Chen, H. Xu, and K. L. Teo, "Stability analysis of a class of nonlinear discrete systems with impulsive effects," Dynamics of Continuous, Discrete \& Impulsive Systems B: Applications \& Algorithms, vol. 17, no. 2, pp. 229-238, 2010.

[10] K. Gu, "Survey on recent results in the stability and control of time delay systems," Journal of Dynamic Systems, Measurement and Control, vol. 125, pp. 158-165, 2003.

[11] X. Li and C. E. de Souza, "Delay-dependent robust stability and stabilization of uncertain linear delay systems: a linear matrix inequality approach," IEEE Transaction of Automatic Control, vol. 42 , no. 8, pp. $1144-1148,1997$.
[12] J. C. Doyle, K. Zhou, and K. Glover, Robust and Optimal Control, Prentice Hall, Upper Saddle River, NJ, USA, 1996.

[13] X. Li and C. E. de Souza, "Criteria for robust stability and stabilization of uncertain linear systems with state delay," Automatica, vol. 33, no. 9, pp. 1657-1662, 1997.

[14] P. Gahinet, A. Nemirovskii, A. J. Laub, and M. Chilali, LMI Control Toolbox for Use with MATLAB, The Math Works, Inc, 1995.

[15] S. Boyd, L. El Ghaoui, E. Feron, and V. Balakrishnan, Linear Matrix Inequalities in System and Control Theory, Society for Industrial and Applied Mathematics (SIAM), Philadelphia, Pa, USA, 1994. 


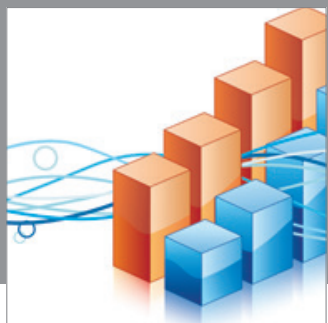

Advances in

Operations Research

mansans

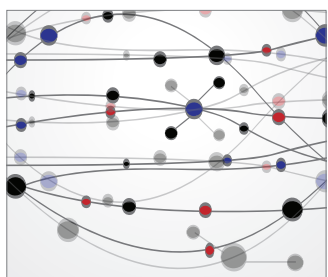

The Scientific World Journal
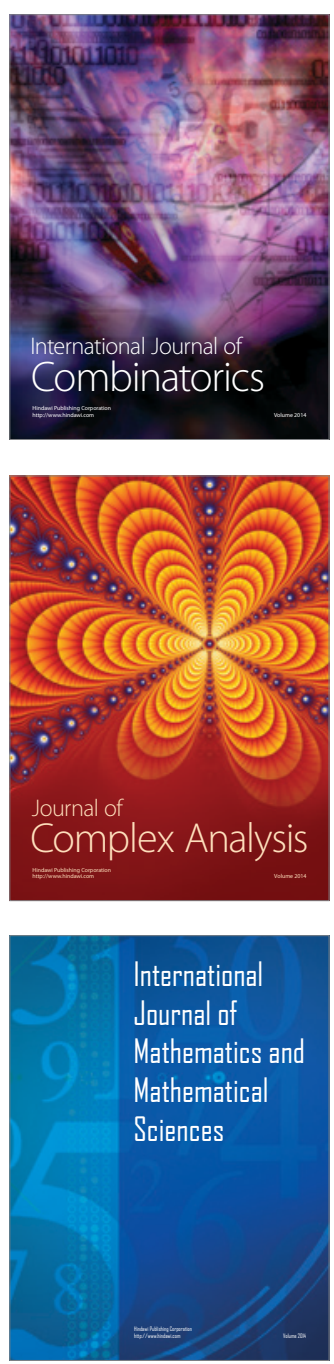
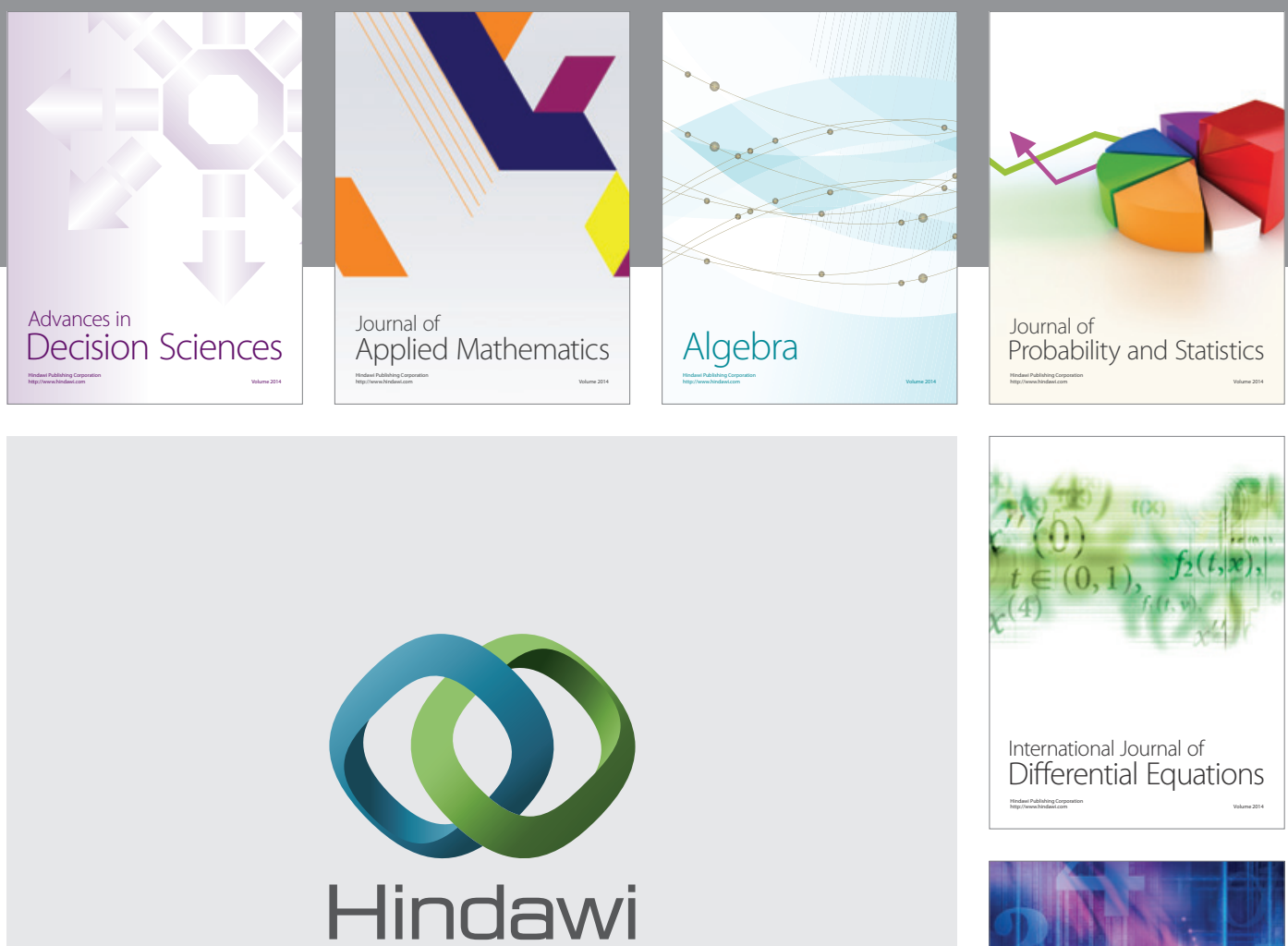

Submit your manuscripts at http://www.hindawi.com
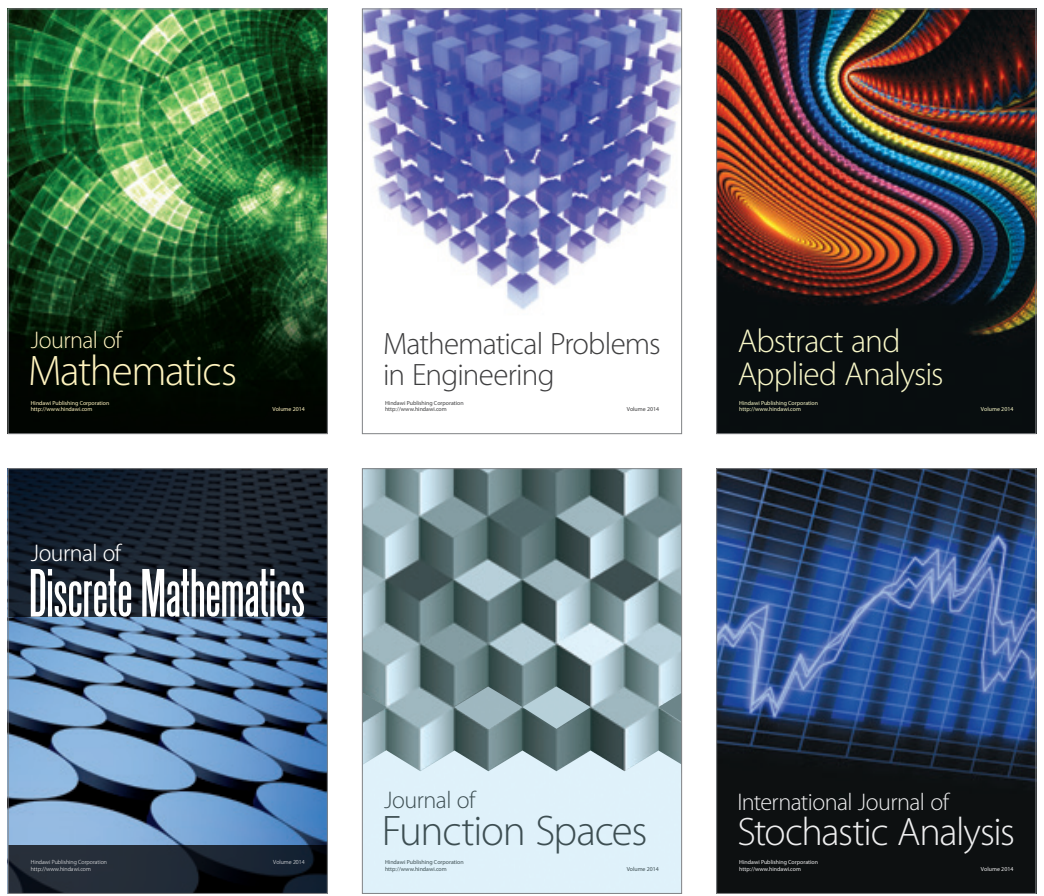

Journal of

Function Spaces

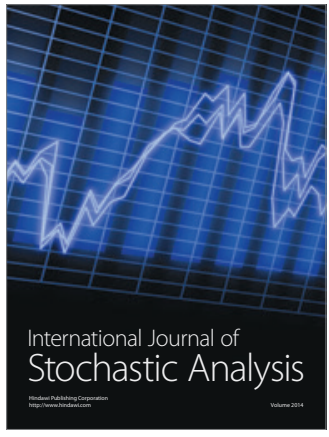

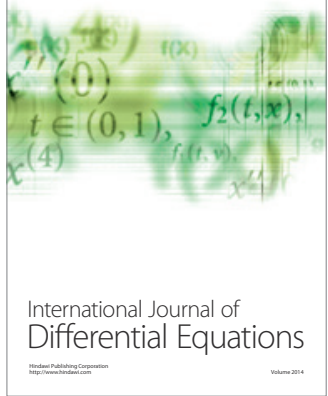
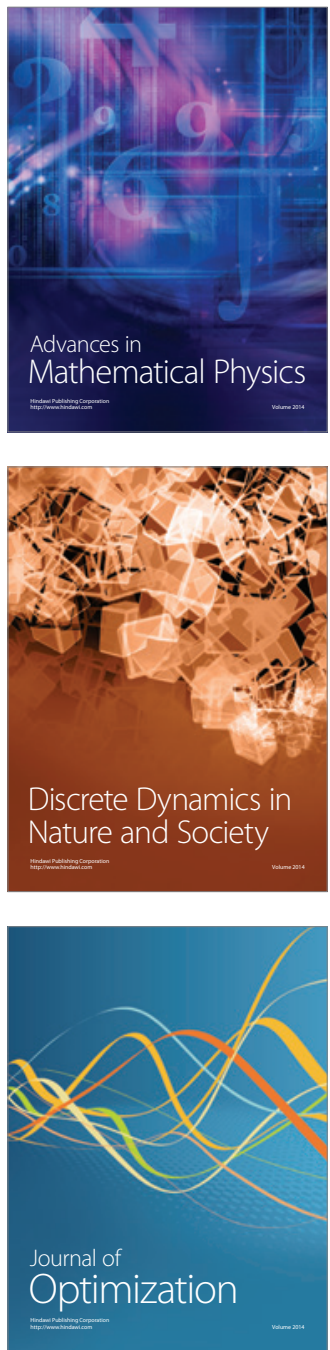